\title{
EDITORIAL
}

\section{Malrotation: some answers but more questions}

\author{
Thomas L. Slovis • Peter J. Strouse
}

Received: 13 January 2009 /Accepted: 14 January 2009/Published online: 25 February 2009

(C) Springer-Verlag 2009

The historical review by Lampl et al. [1] helps us to understand our continual conundrum about the diagnosis and misdiagnosis of malrotation. When a child has obvious malrotation (Table 1), the diagnosis is easy. However, when there are minor deviations of the position of the duodenaljejunal junction on the upper gastrointestinal examination, the correct diagnosis becomes more difficult and is the cause of confusion. Why, in those with no abnormality found at surgery or with an unremarkable follow-up, may the duodenal-jejunal junction be lower than it should be and not reach the level of the duodenal bulb? Why, in those with no abnormality found, may the duodenal-jejunal junction be more midline and not cross the spine entirely? If the "ligament of Treitz" is truly a ligament (fibrous, nonstretchable structure), this should not occur. However, Lampl et al. [1] point out that this ligament is not only a ligament but also a muscle - the original description by Václav Treitz. When one goes to Gray's Anatomy [2], we find "The ligament of Treitz may contain a small slip of muscle, the suspensory muscle of the duodenum. When present, the suspensory muscle contains skeletal muscle fibres that run from the left crus of the diaphragm to connective tissue around the coeliac axis, and smooth muscle fibres that run from the coeliac access: its function

T. L. Slovis $(\bowtie)$

Department of Pediatric Imaging,

Children's Hospital of Michigan,

3901 Beaubien Blvd.,

Detroit, MI 48201, USA

e-mail: pedradeditor@med.wayne.edu

P. J. Strouse

Department of Radiology, Section of Pediatric Radiology,

C.S. Mott Children's Hospital,

University of Michigan Health System,

Ann Arbor, MI, USA is unknown." Obviously, muscle can stretch. When there is dilatation of the stomach, colon, or small bowel, the duodenal-jejunal junction may be pushed downward and/ or toward the midline. The "stretchability" of the ligament of Treitz helps to explain the seeming malposition of the duodenal-jejunal junction in some normal patients.

The paper by Lampl et al. [1] does not detail how the "suspensory muscle" described by Václav Treitz became the "ligament of Treitz," but it does push us toward a rational conclusion as to why these normal variants occur. In our experience, the minor variations in the position of the duodenal-jejunal junction on the frontal projection when the lateral descent and ascent are appropriate have never been associated with a volvulus or progressive symptoms. Now, we have a reasonable explanation why!

The second major point of the article by Lampl et al. is that when one is uncertain that the duodenal-jejunal junction is normal, and there is no clinical reason to go immediately to the operating room, one should follow the contrast agent to find the position of the cecum. In fact, this is merely suggesting reverting to the pre-1960 era when the barium enema was the primary test for malrotation. A small percent of patients with malrotation (and in some cases, volvulus), however, will have a normal cecal position [3]. Lampl et al. and previous authors suggest that the cecal position is useful in showing the distal point of attachment of the midgut mesentery. In both patients with a normal situs and those with heterotaxia, some surgeons have used the knowledge of the proximal attachment point (the duodenal-jejunal junction) and the distal attachment point (cecum) of the mesentery to determine the breadth of the mesenteric root and thus the relative risk for development of volvulus.

Making an assumption of risk based on cecal position, however, is controversial. First, normal cecal position does 
Table 1 Normal radiographic findings of the duodenal-jejunal junction on an upper gastrointestinal examination.

\begin{tabular}{|c|c|}
\hline Frontal view & Lateral view \\
\hline $\begin{array}{l}\text { The duodenal-jejunal junction is as cephalic as the duodenal } \\
\text { bulb }\end{array}$ & $\begin{array}{l}\text { The second portion of the duodenum descends posteriorly in the } \\
\text { retroperitoneum }\end{array}$ \\
\hline The duodenal-jejunal junction is to the left of the spine & $\begin{array}{l}\text { The fourth portion of the duodenum ascends posteriorly in the retroperitoneum } \\
\text { just anterior to the descent of the second portion of the duodenum }\end{array}$ \\
\hline
\end{tabular}

not exclude malrotation. While cecal position can be confirmatory of malrotation, it can never exclude the diagnosis. Second, images show cecal position, not cecal fixation. A malfixated cecum that is positioned in a relatively anatomic position may mislead one to think that the mesenteric root is broader than it truly is. The literature is littered with reports of patients with relatively benign forms of malrotation who developed volvulus. There are no findings relative to the cecum that remove all risk of volvulus.

Finally, is it time to reassess the consequences of overdiagnosis of malrotation versus missing the diagnosis? Failing to properly diagnose and treat a child with malrotation can be catastrophic. In 2009, complications of surgical exploration are rarely catastrophic. The morbidity of surgical exploration using laparoscopy is considerably less than open explorations of years past. In equivocal cases and in positive cases considered "low risk for volvulus," is it really better to wait and watch rather than operate, definitively determine the anatomy and remove any risk of volvulus? The conundrum continues ...

\section{References}

1. Lampl B, Levin TL, Berdon W et al (2009) Malrotation and midgut volvulus: a historical review and current controversies in diagnosis and management. Pediatr Radiol 39: doi:10.1007/s00247-0091168-y

2. Borley NR, Healy JC (2008) Abdomen and pelvis. Section 8. In: Stranding S (ed) Gray's Anatomy, 40th edn. Elsevier, London, UK, p 1127

3. Slovis TL, Klein MD, Watts FB Jr (1980) Incomplete rotation of the intestine with a normal cecal position. Surgery 87:325-330 